Supporting Information

\title{
Characterization of the aryl hydrocarbon receptor (AhR) pathway in Anabas testudineus and mechanistic exploration of the reduced sensitivity of AhR2a
}

Wanglong Zhang ${ }^{1,2}$, Heidi Qunhui Xie ${ }^{1,2}$, Yunping Li ${ }^{1,2}$, Xianghui Zou ${ }^{3}$, Li Xu ${ }^{1,2}$, Dan Ma ${ }^{1,}$

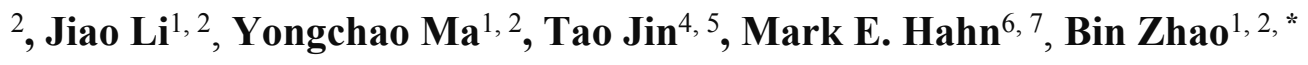

1 State Key Laboratory of Environmental Chemistry and Ecotoxicology, Research Center for Eco-

Environmental Sciences, Chinese Academy of Sciences, Beijing 100085, China

2 University of Chinese Academy of Sciences, Beijing 100049, China

3 School of Food Engineering and Biotechnology, Hanshan Normal University, Chaozhou 521041,

China

4 China National Genebank-Shenzhen, BGI-Shenzhen, Shenzhen 518083, China

5 BGI-Qingdao, Qingdao 266510, China

6 Biology Department, Woods Hole Oceanographic Institution (WHOI), Woods Hole, MA 02543, USA

7 Boston University Superfund Research Program, Boston University, Boston, MA 02118, USA

*Address correspondence to Dr. Bin Zhao, Research Center for Eco-Environmental Sciences (RCEES),

Chinese Academy of Sciences, Beijing 100085, China; Telephone: (86) 010-62842867; Email:

binzhao@rcees.ac.cn

The number of pages: 7

The number of figures: 4

The number of tables: 2 
Figures

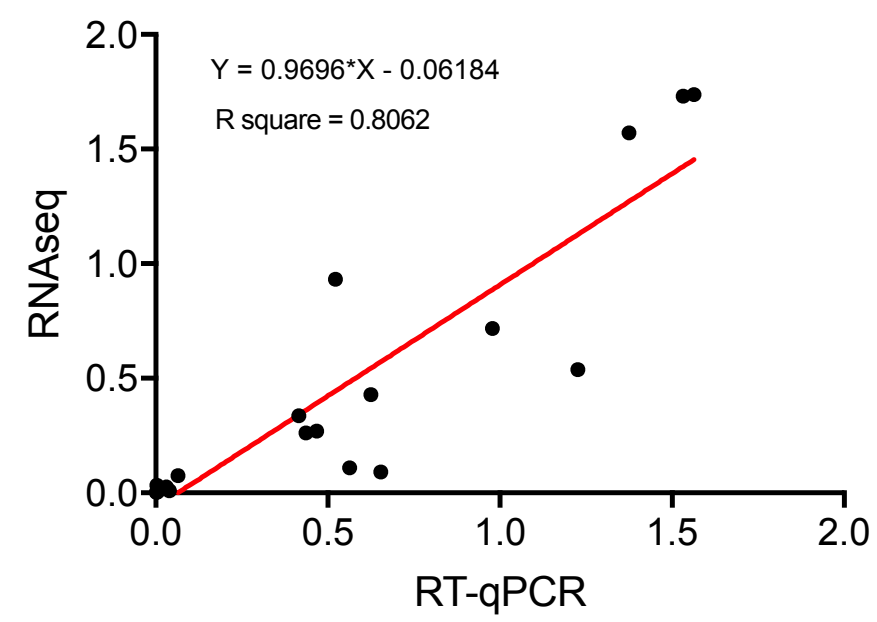

Figure S1. Validation of the RNAseq results using RT-qPCR. The linear regression was performed using a combination of expression data of genes of A. testudineus AhR pathway in the liver, kidney, and gill. 

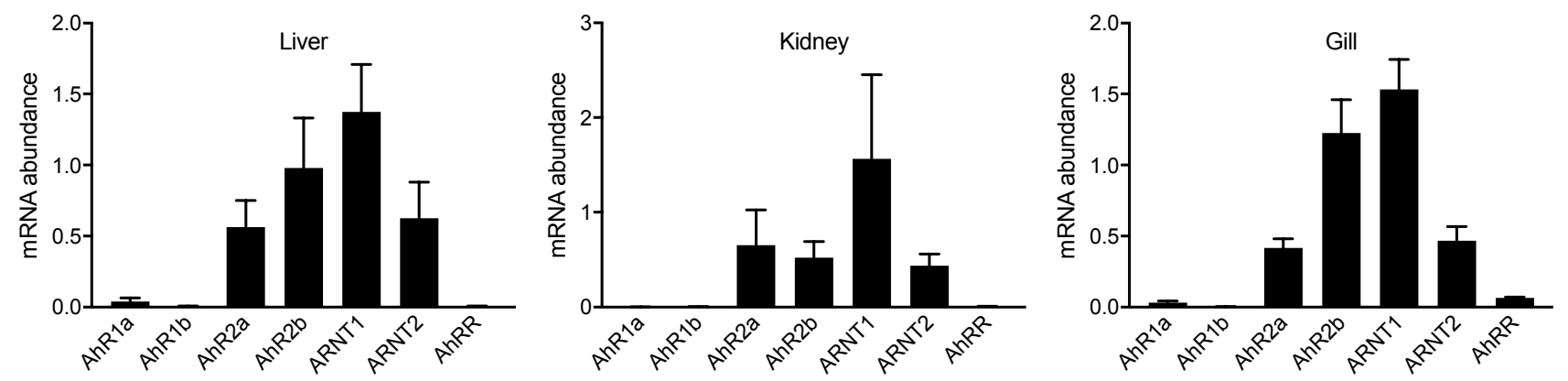

Figure S2. MRNA abundances of AhR pathway members, respectively, in the liver, kidney, and gill of $A$. testudineus measured by RNAseq. 


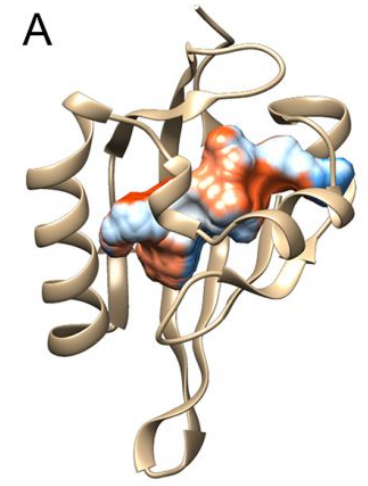

A. testudineus AhR2a

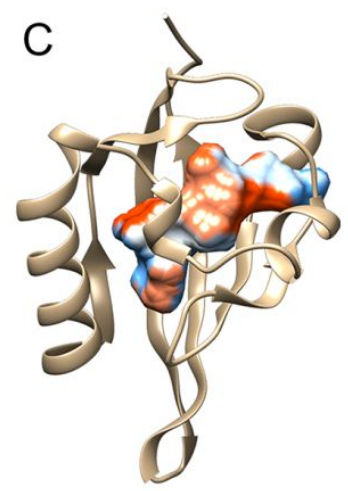

O. latipes AhR2b

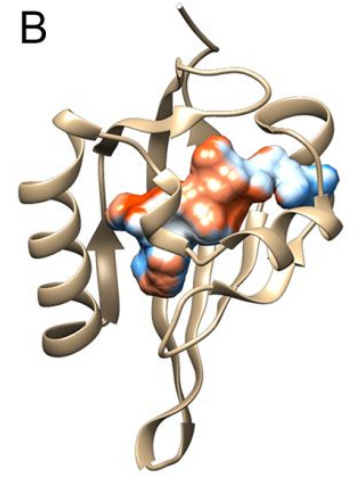

A. testudineus AhR2b

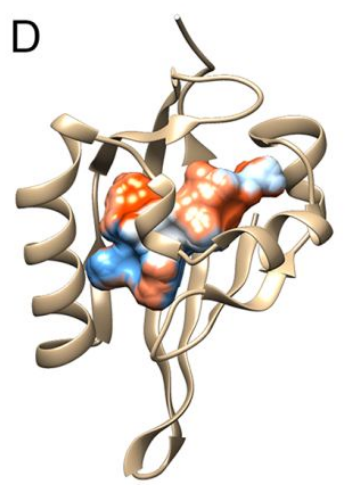

D. rerio $A h R 2 b$

Figure S3. The homology model and ligand binding cavity of $A$. testudineus AhR2a (A), A. testudineus AhR2b (B), O. latipes AhR2b (C), and D. rerio AhR2b (D). Residues forming each cavity are listed in Fig. 4A. 

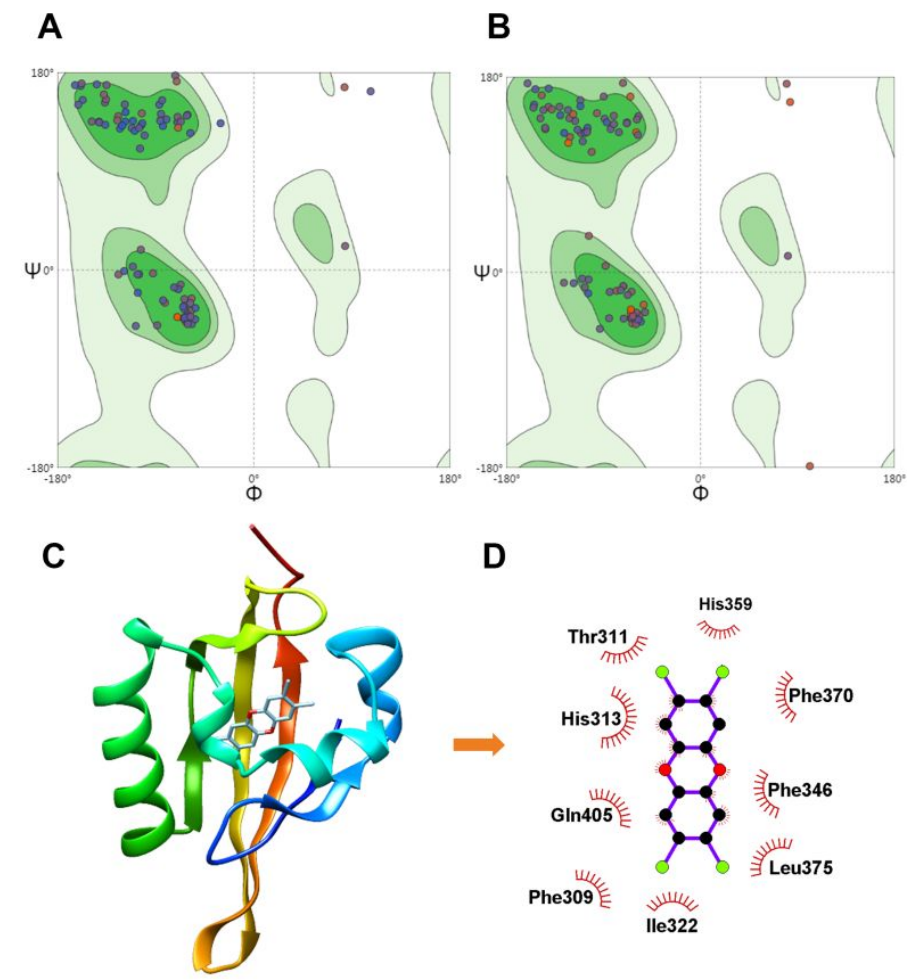

Figure S4. The A. testudineus AhR2a (A) and A. testudineus AhR2b (B) homology models had 93.5\% and $95.4 \%$ residues, respectively, in the most favored areas of the Ramachandran plot. $\mathbf{C}$. The interaction model of the AhR2b LBD with TCDD analyzed by the AutoDock Vina. D. Residues of the AhR2b LBD that interact with TCDD were visualized by the LigPlot+. This information was also simplified and shown in Fig. 4A. 


\section{Tables}

Table S1. RT-qPCR primers used for relative quantification of mRNA expression levels of genes involved in AhR pathway.

\begin{tabular}{llll}
\hline Gene & Forward primer & Reverse primer & Efficiency \\
\hline$\beta$-actin & AGCCAACAGGGAGAAGATGA & GCAGAGCGTAACCCTCGTAG & 95.63 \\
AhR2b & AAGCTTCTTTAACGCCACCA & CCCTCAGAGAAGCTGACACC & 100.45 \\
AhR2a & TGCAGGGTGGTGGTAATACA & CCGTTGTCCCTCAAAACACT & 95.76 \\
AhR1a & GACAAGCTCTCCATCCTTCG & CAGCAGAAGTTCCCCTTCAG & 97.49 \\
AhR1b & GCAAAACCAAAAGCAGCAA & GCTCCCAGCAGGGACTTAG & 98.87 \\
ARNT1 & CAACACCAACGGCTATGATG & GGGAACATGTCTTGCTGGTT & 94.62 \\
ARNT2 & AGTCCTCCATGAGGATGTGC & AGCCTCTCCTTCCTTTGAGG & 95.74 \\
AhRR & GTCTGCAAACCAGGAGAACC & AGAATCCAGACGTGCTGTCC & 96.85 \\
\hline
\end{tabular}


Table S2. Primers for site-directed mutagenesis.

\begin{tabular}{|c|c|}
\hline Primer & Sequence (5' - 3') \\
\hline AhR2a-flag-cds-forward & TGTGGTGGAATTCTGCAGATGCCACCATGGACTACAAGGACGACGATGACAAGAGTGACACCATGTTGGGG \\
\hline AhR2a-cds-reverse & CGGCCGCCACTGTGCTGGATTCAGCTTAGCTAGACTCGGCTCTACTCC \\
\hline AhR2a-mut1-linker-reverse & GCCAAGAACAATCTTCCCTCTCGTGTCGATGCCCGTGGGTGTGAAGTCCA \\
\hline AhR2a-mut1-linker-forward & AGAGGGAAGATTGTTCTTGGC \\
\hline AhR2a-mut2-linker-reverse & GATGAACTGGTACCCAGAGCCCCTCATACACAGCTCGATCTCTG \\
\hline AhR2a-mut2-linker-forward & GGCTCTGGGTACCAGTTCATC \\
\hline AhR2a-mut3-linker-reverse & TCAGGAGCCTGAAAACAGTGAATCCGCTCTCTCCTGTTTTAA \\
\hline AhR2a-mut3-linker-forward & ACTGTTTTCAGGCTCCTGA \\
\hline AhR2a-mut4-linker-reverse & TGTAGATCAGCTTGGCGTTGGACTGCACCCACACCCAGCCAG \\
\hline AhR2a-mut4-linker-forward & AACGCCAAGCTGATCTACA \\
\hline AhR2b-flag-cds-forward & TGTGGTGGAATTCTGCAGATGCCACCATGGACTACAAGGACGACGATGACAAGCGCTTCAACATGCTGTCG \\
\hline AhR2b-cds-reverse & CGGCCGCCACTGTGCTGGATTCAGCTTAGCTATTTGTTCTCTGAGTGGTATGGGTTCCCA \\
\hline AhR2b-mut1-linker-reverse & AACCTAGAACCACCTTCCCTCTGCTACAAACGCCTGTTGGTGTGAAGTCCA \\
\hline AhR2b-mut1-linker-forward & AGAGGGAAGGTGGTTCTAGGTT \\
\hline AhR2b-mut2-linker-reverse & GATGAAGTGGTAGCCTGAACCCTTCATACAGAGTTCTATCTCGT \\
\hline AhR2b-mut2-linker-forward & GGTTCAGGCTACCACTTCATC \\
\hline AhR2b-mut3-linker-reverse & GTCAGCAGTCTGAAGACAGTCATGCCGCTCTCTCCAGTTTTTA \\
\hline AhR2b-mut3-linker-forward & ACTGTCTTCAGACTGCTGAC \\
\hline AhR2b-mut4-linker-reverse & TTAAAGACCAGCCTGGCATTTGACTGCACCCAGACCCATGTCC \\
\hline AhR2b-mut4-linker-forward & AATGCCAGGCTGGTCTTTAA \\
\hline
\end{tabular}

